\title{
Grading in Myxofibrosarcoma of the Extremities Can Predict Survival and Local Control
}

\author{
Andrea Sambria, Gianmarco Tuzzato ${ }^{a}$ Paolo Spinnato $^{\mathrm{a}}$ \\ Massimiliano De Paolis ${ }^{c}$ Davide Maria Donati ${ }^{a}{ }^{a}$ Giuseppe Bianchi ${ }^{a}$

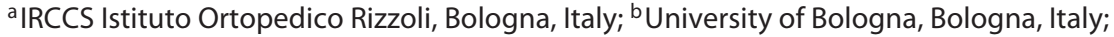 \\ 'Azienda Ospedaliera Sant'Orsola-Malpighi, Bologna, Italy
}

\section{Keywords}

Myxofibrosarcoma · Prognosis · Histologic grade

\begin{abstract}
Background: The aim of this retrospective analysis is to understand the natural history of myxofibrosarcoma (MFS), in particular whether the prognosis can be influenced by histologic grade. Methods: We reviewed 229 adult patients with primary MFS of the limbs. We analyzed disease-specific survival (overall survival [OS]) and local recurrence (LR). Results: Median age was 70 years (range, 19-92). Sixteen (7.0\%) were grade 1, $38(16.6 \%)$ grade 2, and 175 (76.4\%) grade 3. A worse OS was found in grade 3 MFS (73.1\%) than in grade 2 and 1 MFS (91.9 and $100 \%$, respectively) at 5 years $(p=0.031)$. Locally recurred MFS had a worse OS $(p=0.018)$. A better LRfree rate ( $100 \%$ at 5 years) was observed in grade 1 MFS; however, a similar rate was observed between grade 2 and 3 tumors (77.1 and $80.0 \%$ at 5 years, respectively, $p=0.412$ ). Conclusions: Grade 3 MFS has the worst prognosis. Grade 1 MFS have the lowest risk of LR. These data could help identify a high-risk patient group, thus selecting a more careful follow-up for higher-risk patients. Since MFS mostly affects the elderly population, it might be useful to reserve adjuvant treatments (radiotherapy and chemotherapy) to higher-risk patients.

○) 2020 S. Karger AG, Basel
\end{abstract}

\section{Introduction}

Myxofibrosarcoma (MFS) is a soft-tissue sarcoma (STS), which primarily affects the extremities and girdles, especially in elderly patients [1].

The local recurrence (LR) rate is particularly high in this sarcoma (range 32-60\%) [2, 3], superior to other STS, even in the case of wide excision [4]. A possible explanation for the high LR rate is the characteristically infiltrative pattern of the tumor that can manifest itself in MRI as a curvilinear shape, commonly defined as "tail," which extends from the primary mass in surrounding soft tissues [5-8].

Thus, differently from other STS, wide-margin assessment does not correlate with lower recurrence rate [9, 10]. It remains unclear whether standard margin assessment is adequate for MFS $[11,12]$.

The role of radiation therapy (RT) is also still debated, because it is actually challenging to enclose all potential residual tumor into the RT field. Moreover, MFS is supposed to be a radioresistant sarcoma $[13,14]$.

A high percentage (15-38\%) of locally recurrent MFSs progress to a higher-grade disease with an attendant increase in metastatic potential [1]. Nevertheless, the prognosis of MFS is relatively good compared to other STS, with a risk of distant metastases (DM) ranging between 20 and 25\% [2] and a disease-specific survival (overall survival [OS]) of $83 \%$ [13]. 
Table 1. Patients' characteristics at baseline according to FNLCC grade

\begin{tabular}{|c|c|c|c|c|c|}
\hline Characteristics & $\begin{array}{l}\text { Grade 1 } \\
(n=16)\end{array}$ & $\begin{array}{l}\text { Grade } 2 \\
(n=38)\end{array}$ & $\begin{array}{l}\text { Grade 3 } \\
(n=175)\end{array}$ & Overall & $\begin{array}{l}p \\
\left(\chi^{2} \text { test }\right)\end{array}$ \\
\hline Median age (range), years & $65(44-80)$ & $68(21-92)$ & $70(18-92)$ & $70(19-92)$ & 0.802 \\
\hline \multicolumn{6}{|l|}{ Sex } \\
\hline Male & $4(25.0$ & $25(65.8)$ & $88(50.3)$ & $117(51.1)$ & \multirow[t]{2}{*}{0.021} \\
\hline Female & $12(75.0)$ & $13(34.2)$ & $87(49.7)$ & $112(48.9)$ & \\
\hline \multicolumn{6}{|l|}{ Localization } \\
\hline Lower limbs & $9(56.3)$ & $27(71.1)$ & $127(72.6)$ & $163(71.2)$ & \multirow[t]{4}{*}{0.209} \\
\hline Upper limbs & $0(0)$ & $7(18.4)$ & $23(13.1)$ & $30(13.0)$ & \\
\hline Pelvic girdle & $3(18.8)$ & $3(7.9)$ & $12(6.9)$ & $18(7.9)$ & \\
\hline Shoulder girdle & $4(25.1)$ & $1(2.6)$ & $13(7.4)$ & $18(7.9)$ & \\
\hline \multicolumn{6}{|l|}{ Size } \\
\hline$<5 \mathrm{~cm}$ & $5(31.3)$ & $15(39.5)$ & $20(11.4)$ & $40(17.5)$ & \multirow[t]{3}{*}{0.036} \\
\hline $5-10 \mathrm{~cm}$ & $9(56.3)$ & $12(31.6)$ & $64(36.6)$ & $85(37.1)$ & \\
\hline$>10 \mathrm{~cm}$ & $2(12.4)$ & $11(28.9)$ & $91(52.0)$ & $104(45.4)$ & \\
\hline \multicolumn{6}{|l|}{ Depth } \\
\hline Superficial & $3(18.8)$ & $11(28.9)$ & $29(16.6)$ & $43(18.8)$ & \multirow[t]{2}{*}{0.209} \\
\hline Deep & $13(81.2)$ & $27(71.1)$ & $146(83.4)$ & $186(81.2)$ & \\
\hline
\end{tabular}

Values are presented as $n(\%)$, unless otherwise indicated.

Overall, tumor grade correlates with the rate of metastases and tumor-related death $[13,14]$. However, none of the previous studies analyzed a homogeneous cohort of patients affected by primary MFS of the extremities. In an effort to improve the current understanding of the natural history and treatment outcomes of primary localized MFS of the extremities, we conducted a retrospective analysis of patients treated at a single institute.

\section{Materials and Methods}

A total of 384 patients affected by MFS of the limbs (hand and foot excluded) underwent surgical excision at a single institute between 1993 and 2016. This research has been approved by the IRB of the authors' affiliated institutions.

Patients were excluded if they presented a LR $(n=32)$ or if they had a previous unplanned excision $(n=98)$ [15]. Twenty-five patients were accordingly excluded because they presented metastasis at the time of diagnosis. In total, 229 adult patients ( $>18$ years) with primary and localized MFS of the extremities (hand and foot excluded) were included in the study. All cases were histologically revised and classified according to the 2013 World Health Organization classification of STS [16] by experienced sarcoma pathologists of our Institute.

A 3-step system (FNCLCC) was used to assess MFS grades [17]. The tumor size was assessed on surgical specimens using the larger diameter as a reference, while depths were divided into superficial (above the fascia) and deep (below the fascia), according to preoperative MRI. Margins were evaluated according to the Enneking classification [18]. Radical and wide margins were considered "adequate."

The use of RT and chemotherapy (ChT) was decided collegially with a multidisciplinary team (orthopedic surgeon, radiotherapist, and oncologist). Patients' characteristics were presented by frequencies and percentages for categorical variables, median and range for continuous variables. The Kaplan-Meier method was used to estimate OS and LR rates. LR-free survival was defined as the time between surgery and the first LR, respectively, or last follow-up available, whichever came first. Similarly, OS interval was defined as the time between surgery and death or last follow-up, whichever came first. Patients who died of other causes were censored. Differences in survival rates were assessed by the log-rank test.

All analyses were completed using the Statistical Package for Social Science (IBM Corp. Released 2013. IBM SPSS Statistics for Windows, Version 22.0; IBM Corp., Armonk).

\section{Results}

Median age at the time of surgery was 70 years (range 19-92); 117 patients $(51.1 \%)$ were male, 112 (48.9\%) were female. The majority of MFS $(163,71.2 \%)$ were localized in the lower limbs, 30 (13.0\%) in the upper limbs, 18 (7.9\%) in the pelvic girdle, and $18(7.9 \%)$ in the shoulder girdle. Forty tumors $(17.5 \%)$ were small $(<5 \mathrm{~cm}), 85(37.1 \%)$ between 5 and $10 \mathrm{~cm}$, and $104(45.4 \%)$ were $>10 \mathrm{~cm}$. Forty-three MFS (18.8\%) were superficial, and $186(81.2 \%)$ were deep (Table 1). Sixteen (7.0\%) MFS were grade 1, 38 (16.6\%) grade 2 , and $175(76.4 \%)$ grade 3 (Table 1). Higher-grade tumors were more often larger $(p=0.036)$, while the depth of MFS was not related to histologic grade $(p=0.209)$. In $213(93.0 \%)$ patients excision was possible, whereas in 16 (7.0\%) patients amputation was necessary.

Out of the excised MFS, 172 (82.1\%) had adequate (wide) margins and 41 (17.9\%) inadequate margins (marginal in 32 cases and intralesional in 9 cases). Overall, 150 patients $(65.5 \%)$ received adjuvant or neoadjuvant RT as complementary treatment to surgery. It was 


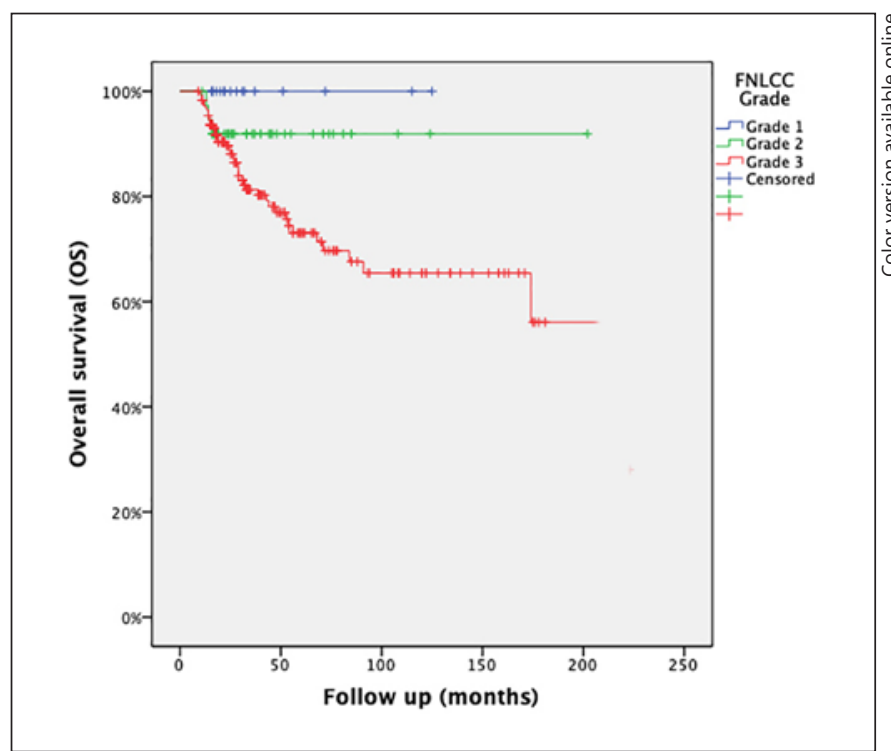

Fig. 1. Kaplan-Meier OS curve according to FNLCC tumor grade.

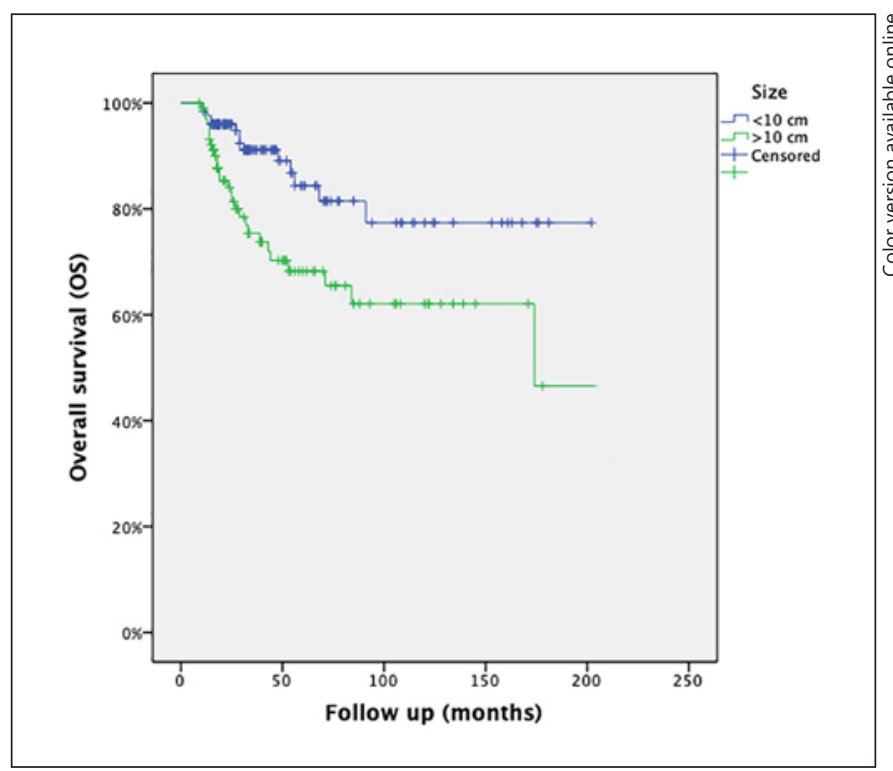

Fig. 3. Kaplan-Meier OS curve according to tumor size.

mostly used in deep ( $p=0.013)$ MFS, particularly after inadequate surgical margins $(p=0.002)$. ChT was administered to $47(20.5 \%)$ patients (median age 61 years, range 19-76), in particular in MFS $>10 \mathrm{~cm}(p=0.012)$, deep tumors $(p<0.001)$, and in patients with grade 3 MFS $(p=0.001)$.

At the last follow-up (median 33 months, range 9-198), 149 patients (65.1\%) were alive with no evidence of disease, $10(4.4 \%)$ were alive with progression of the disease, 27 patients (11.8\%) died of other causes, and $43(18.8 \%)$ died of the disease. Kaplan-Meier analysis with OS as a primary endpoint showed an estimated survival of $77.1 \%$

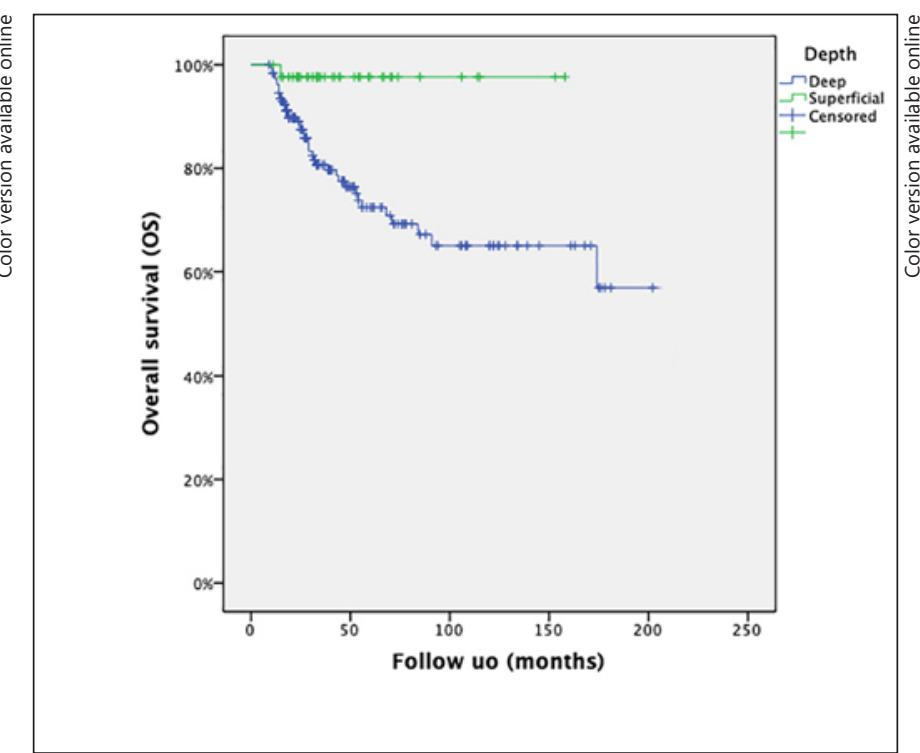

Fig. 2. Kaplan-Meier OS curve according to tumor depth.

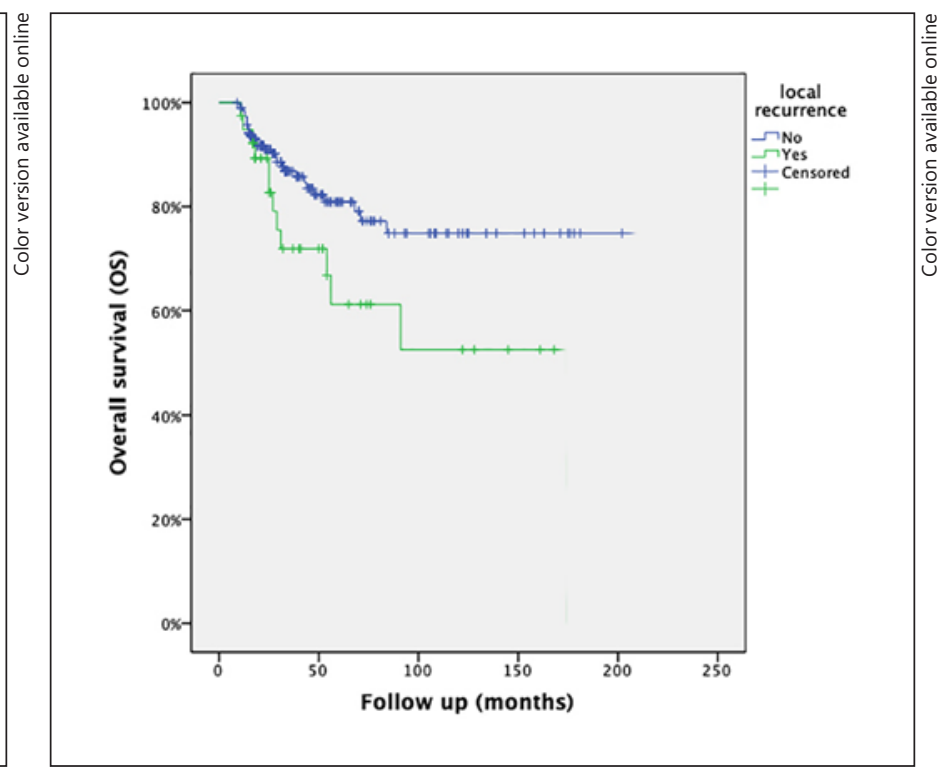

Fig. 4. Kaplan-Meier OS curve according to LR.

(CI 95\% 70.1-84.1) at 5 years and 70.4\% (CI 95\% 61.8$79.0)$ at 10 years. A worse OS was found in grade 3 MFS (73.1\%), compared to grade 2 and grade 1 MFS (91.9 and $100 \%$, respectively, at 5 years, $p=0.031$ ) (Fig. 1). A better OS was observed in superficial MFS ( 97.6 vs. $72.4 \%$ at 5 years, 97.6 vs. $65.0 \%$ at 10 years, $p=0.005$ ) (Fig. 2), while MFS $>10 \mathrm{~cm}$ had a worse OS (68.2 vs. $84.4 \%$ at 5 years, and 62.1 vs. $77.4 \%$ at 10 years, $p=0.002$ ) (Fig. 3). Locally recurred MFS, considered a time-dependent covariate, had a worse OS ( 80.9 vs. $61.2 \% p=0.018)$ (Fig. 4).

LR was observed in 39 patients (17.0\%) after a median 18 months (range 3-133). A LR-free rate of $80.9 \%$ (CI 


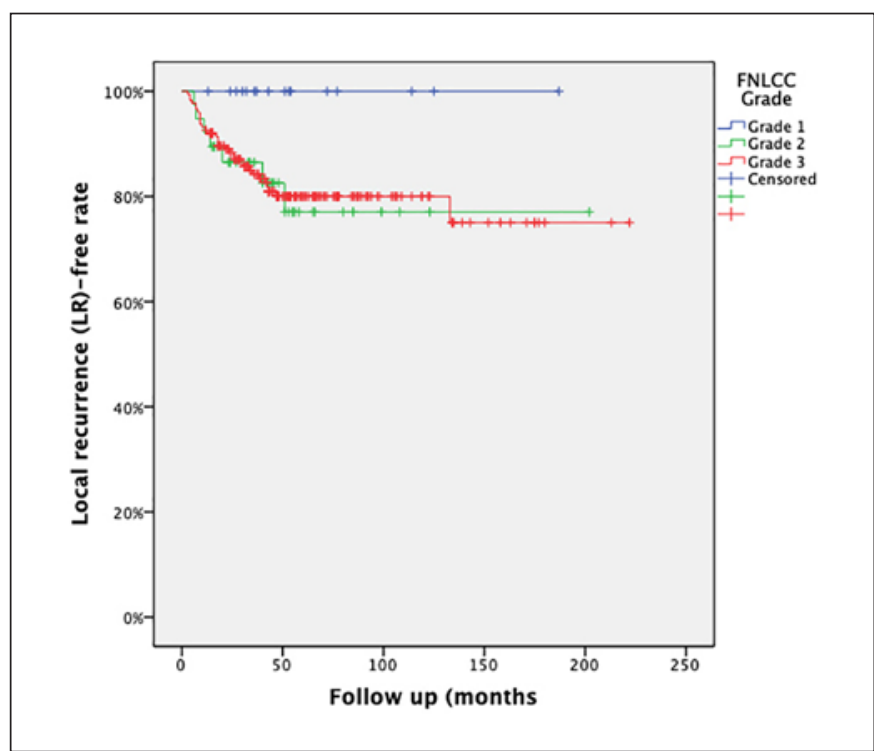

Fig. 5. Kaplan-Meier LR curve according to FNLCC tumor grade.

$95 \% 75.2-86.6)$ at 5 and 10 years was found.A similar rate was observed between grade 2 and grade 3 tumors (77.1 and $80.0 \%$ at 5 years, respectively, $p=0.412$ ) (Fig. 5). However, no LR was observed in grade 1 MFS (LR-free survival $100 \%$ at 5 years).

No differences in the LR rate were observed between patients with adequate and inadequate surgical margins ( $p=0.876)$. However, none of the patients who had radical margins developed a LR.

\section{Discussion}

Tumor grading was suggested to predict the risk of developing DM, but not the local failure in MFS [13]. However, the existing studies only investigated the role of FNLCC grading in a heterogeneous cohort of patients (extremities and trunk/neck and primary/recurrent MFS) [13]. To the best of our knowledge, this is the first study in which primary, localized MFS of the extremities are evaluated separately.

In the present series, the OS was correlated to the histological tumor grade. As far as the tumor grade is concerned, the main difference in outcome was observed between grade 2 and grade 3 tumors, while less relevant differences were identified between grade 1 and 2 MFS. None of the patients affected by grade 1 MFS died of the disease. On the other hand, the mortality rate of patients affected by grade 3 MFS was similar to that of other histotypes affecting the extremities. Our findings differ from those of Sanfilippo et al. [13] in whose series different anatomic regions were included. It is known that STS behavior can be extremely different according to anatomic region, such as extremities, neck, and trunk [14]. In addition, locally recurred MFS had a worse OS (80.9 vs. $61.2 \%)$. This may also explain our different findings with respect to those of Sanfilippo et al. [13], which included both primary and recurrent MFS. In LR, a progression of grade may eventually be observed, thus increasing mortality. As expected, a significantly better OS was also observed in superficial MFS and in MFS $<10 \mathrm{~cm}$.

LR was observed in $17 \%$ of the patients after a median of 18 months, with a LR-free rate of $80.9 \%$ at 5 and 10 years. The incidence of LRs was definitely higher than that of all other STSs (approximately $10 \%$ ) $[2,14]$. LR rate remained stable both in the medium- and long-term follow-up. This emphasizes the need for a strict local control, especially during the first 5 years.

None of the patients affected by grade 1 MFS developed a LR; on the other hand, a similar rate of LR was observed between grade 2 and 3 myxofibrosarcoma.

No differences in the LR rate were observed regarding the adequacy of surgical margins. However, none of the patients who had radical margins later developed a LR [19]. These results empower the findings of Fujiwara et al. [11] who demonstrated that in MFS, a $10-\mathrm{mm}$ excess margin was associated with the lowest risk of LR. Still, the infiltrative growth pattern observed in MFS can make it difficult to highlight the real extent of the tumor in preoperative, intraoperative, and postoperative settings (specimen examination) $[5,7,11]$.

In our series, RT seemed to have no real impact on reducing the risk of a LR. However, for obvious reasons, patients who received RT were unfavorably selected, the choice being a clinical one performed on an individual basis. Thus, it can be argued that the similar LR rate in the RT and non-RT groups might imply an effect [20].

There is a limitation to the present work that must be acknowledged, and that is its being a retrospective study. Nevertheless, we report data from a selected cohort of patients including only adult patients affected by primary, localized MFS of the extremities. To the best of our knowledge, this is the first series of such a number of cases with homogenous characteristics that analyzes the effects of grading in MFS.

In conclusion, our findings in a large series of $229 \mathrm{pa}-$ tients affected by primary and localized MFS of the extremities indicate that grade $3 \mathrm{MFS}$ are associated with the worst prognosis. On the other hand, patients affected by grade 1 MFS have the lowest risk of developing a LR.

These data could have important implications for the treatment approach, making it possible to identify a highrisk patient group and thus selecting a more careful follow-up for higher-risk patients. Furthermore, since MFS mostly affects the elderly population, it might be of paramount importance to arrange adjuvant treatments such as RT and ChT only for higher-risk patients. 


\section{Acknowledgements}

We would like to thank Giulia Zoggia for her help in the transcription of the text in English.

\section{Statement of Ethics}

Ethic committee approval is not required for retrospective studies in our Institutions.

\section{Disclosure Statement}

All authors have no conflicts of interest to declare.

\section{Funding Sources}

The authors did not receive any funding.

\section{Author Contributions}

Conceived and designed the analysis: A.S., D.M.D., G.B.

Collected the data: G.T., P.S., M.D.P.

Contributed data or analysis tools: G.B., P.S., G.T.

Performed the analysis: A.S., G.B.

Wrote the paper: A.S., D.M.D., M.D.P.

\section{References}

1 Willems SM, Debiec-Rychter M, Szuhai K, Hogendoorn PC, Sciot R. Local recurrence of myxofibrosarcoma is associated with increase in tumour grade and cytogenetic aberrations, suggesting a multistep tumour progression model. Mod Pathol. 2006 Mar;19(3):407-16.

2 Gronchi A, Lo Vullo S, Colombo C, Collini P, Stacchiotti S, Mariani L, et al. Extremity soft tissue sarcoma in a series of patients treated at a single institution: local control directly impacts survival. Ann Surg. 2010 Mar;251(3) 506-11.

3 Mentzel T, Calonje E, Wadden C, Camplejohn RS, Beham A, Smith MA, et al. Myxofibrosarcoma. Clinicopathologic analysis of 75 cases with emphasis on the low-grade variant. Am J Surg Pathol. 1996 Apr;20(4):391-405.

4 Weiss SW, Enzinger FM. Myxoid variant of malignant fibrous histiocytoma. Cancer. 1977 Apr;39(4):1672-85.

5 Kaya M, Wada T, Nagoya S, Sasaki M, Matsumura T, Yamaguchi T, et al. MRI and histological evaluation of the infiltrative growth pattern of myxofibrosarcoma. Skeletal Radiol. 2008 Dec;37(12):1085-90.

6 Waters B, Panicek DM, Lefkowitz RA, Antonescu CR, Healey JH, Athanasian EA, et al. Low-grade myxofibrosarcoma: CT and MRI patterns in recurrent disease. AJR Am J Roentgenol. 2007 Feb;188(2):W193-8.

7 Sambri A, Spinnato P, Bazzocchi A, Tuzzato GM, Donati D, Bianchi G. Does pre-operative MRI predict the risk of local recurrence in primary myxofibrosarcoma of the extremities? Asia Pac J Clin Oncol. 2019 Oct;15(5):e181-6.
8 Mansoor A, White CR Jr. Myxofibrosarcoma presenting in the skin: clinicopathological features and differential diagnosis with cutaneous myxoid neoplasms. Am J Dermatopathol. 2003 Aug;25(4):281-6.

9 Hermanek P, Wittekind C. The pathologist and the residual tumor (R) classification. Pathol Res Pract. 1994 Feb;190(2):115-23.

10 Rosenberg SA, Tepper J, Glatstein E, Costa J, Baker A, Brennan M, et al. The treatment of soft-tissue sarcomas of the extremities: prospective randomized evaluations of (1) limbsparing surgery plus radiation therapy compared with amputation and (2) the role of adjuvant chemotherapy. Ann Surg. 1982 Sep; 196(3):305-15.

11 Fujiwara T, Stevenson J, Parry M, Tsuda Y, Tsoi K, Jeys L. What is an adequate margin for infiltrative soft-tissue sarcomas? Eur J Surg Oncol. 2020 Feb;46(2):277-281.

12 Sambri A, Bianchi G, Righi A, Ferrari C, Donati D. Surgical margins do not affect prognosis in high grade myxofibrosarcoma. Eur J Surg Oncol. 2016 Jul;42(7):1042-8.

13 Sanfilippo R, Miceli R, Grosso F, Fiore M, Puma E, Pennacchioli E, et al. Myxofibrosarcoma: prognostic factors and survival in a series of patients treated at a single institution. Ann Surg Oncol. 2011 Mar;18(3):720-5.
14 Brennan MF, Antonescu CR, Moraco N, Singer S. Lessons learned from the study of 10,000 patients with soft tissue sarcoma. Ann Surg. 2014 Sep;260(3):416-21; discussion 421-2.

15 Giuliano AE, Eilber FR. The rationale for planned reoperation after unplanned total excision of soft-tissue sarcomas. J Clin Oncol. 1985 Oct;3(10):1344-8.

16 Jo VY, Fletcher CD. WHO classification of soft tissue tumours: an update based on the 2013 (4th) edition. Pathology. 2014 Feb;46(2): 95-104.

17 Trojani M, Contesso G, Coindre JM, Rouesse J, Bui NB, de Mascarel A, et al. Soft-tissue sarcomas of adults; study of pathological prognostic variables and definition of a histopathological grading system. Int J Cancer. 1984 Jan;33(1):37-42.

18 Enneking WF, Spanier SS, Goodman MA. A system for the surgical staging of musculoskeletal sarcoma. Clin Orthop Relat Res. 1980 Nov-Dec;(153):106-20.

19 Sambri A, Bianchi G, Cevolani L, Donati D, Abudu A. Can radical margins improve prognosis in primary and localized epithelioid sarcoma of the extremities? J Surg Oncol. 2018 May;117(6):1204-10.

20 Teurneau H, Engellau J, Ghanei I, Vult von Steyern F, Styring E. High Recurrence Rate of Myxofibrosarcoma: The Effect of Radiotherapy Is Not Clear. Sarcoma. 2019 Oct;2019: 8517371. 